\title{
Mugny Gabriel, Falomir-Pischastor Juan Manuel et Quiamzade Alain : Influences sociales
}

\section{Thomas Heller}

\section{(2) OpenEdition}

\section{Journals}

Édition électronique

URL : https://journals.openedition.org/edc/10511

DOI : $10.4000 /$ edc. 10511

ISSN : 2101-0366

Éditeur

Université de Lille

Édition imprimée

Date de publication : 1 juillet 2020

Pagination : 219-227

ISBN : 978-2-917562-23-9

ISSN : $1270-6841$

Référence électronique

Thomas Heller, « Mugny Gabriel, Falomir-Pischastor Juan Manuel et Quiamzade Alain : Influences sociales », Études de communication [En ligne], 54 | 2020, mis en ligne le 01 juillet 2020, consulté le 08 janvier 2022. URL : http://journals.openedition.org/edc/10511 ; DOI : https://doi.org/10.4000/edc. 10511

Ce document a été généré automatiquement le 8 janvier 2022

(c) Tous droits réservés 


\title{
Mugny Gabriel, Falomir-Pischastor Juan Manuel et Quiamzade Alain : Influences sociales
}

\author{
Thomas Heller
}

\section{RÉFÉRENCE}

Mugny G., Falomir-Pischastor J. M., Quiamzade A. (2017). Influences sociales, Grenoble, Presses universitaires de Grenoble.

1 L'annonce de la parution de cet ouvrage par l'éditeur était introduite par une accroche pour le moins intrigante : «influencer, c'est bien. Se faire influencer c'est mieux ». Que pouvait bien réserver la lecture d'un ouvrage universitaire sur l'influence sociale qui se présente ainsi, dans les termes d'une mystérieuse position morale?

2 D'autant que l'actualité récente fournit des raisons de mettre en doute la pertinence d'une telle affirmation, que l'on songe à la relation entre la propagande de Daech sur le net et le départ de jeunes combattre en Syrie, au développement des fake news, à leur circulation, et à leurs usages stratégiques par un gouvernement pour nuire à la réputation des journalistes, ou encore aux efforts déployés par certaines directions pour que règne le bonheur dans certaines entreprises capitalistes... Certes, la critique suggérée par ces quelques exemples, fondée aussi sur une position morale, est de peu de portée et ne remet pas fondamentalement en cause cette affirmation : question de point de vue (le bien et le mieux renvoyant alors à la question : pour qui ou pour quoi ?).

3 En fait, la formule trouve dans les premières pages du livre quelques éclaircissements : d'une part, les auteurs rappellent le bénéfice qu'un individu peut tirer d'avoir été influencé (influence constitutive d'un apprentissage, d'une évolution...), qui autorise donc un jugement moral positif; et, dans le prolongement, c'est la représentation de l'influenceur qui s'en trouve également positivement modifiée. D'autre part, les auteurs 
rappellent que dans notre société libérale être influencé, ou être influençable, n'est pas une qualité dont on se réclame aisément. C'est qu'une telle caractéristique est peu compatible avec l'idéal d'autonomie et de responsabilité qui définit l'individu. En ce sens, la formule est un pied-de-nez un peu ironique à une certaine image sociale de l'influençabilité.

4 C'est d'ailleurs l'un des intérêts de bien des travaux dans le domaine de la psychologie sociale : égratigner cette figure encombrante de l'individu libéral, et aussi mettre au jour certains mécanismes psycho-sociaux de ce qu'on pourrait appeler la subjectivation libérale (cf. notamment les travaux de Jean-Léon Beauvois ${ }^{1}$ ou de Nicole Dubois ${ }^{2}$ sur l'internalité).

5 Faut-il le rappeler? L'influence est au principe de la communication humaine. " On ne peut pas ne pas influencer ", écrit Paul Watzlawick avant d'ajouter: «Il est donc absurde de se demander comment il serait possible d'éviter toute influence ou manipulation: il ne nous reste plus qu'à accepter la responsabilité inéluctable de décider pour nous-mêmes de quelle manière cette loi fondamentale de la communication humaine peut être suivie le plus humainement, le plus honnêtement et le plus efficacement possible ${ }^{3}$ ». La recherche en psychologie sociale en général et sur l'influence sociale en particulier apporte sa contribution, par un travail de déconstruction de cette loi.

L'ouvrage présenté ici est une proposition de synthèse de travaux et d'avancées relevant de ce domaine de recherche qu'est l'influence sociale. Il est défini comme une actualisation d'un livre paru en 1995 chez le même éditeur (les PUG), dirigé par Gabriel Mugny (qui co-dirige l'édition de 2017), Dominique Oberlé et Jean-Léon Beauvois et intitulé "Relations humaines, groupes et influence sociale». On y trouve aussi la contribution de Juan Manuel Falomir, co-auteur du présent ouvrage. Celui-ci est composé de quatre chapitres.

7 Dans le premier, intitulé «Fondamentaux de l'influence sociale», les auteurs posent une définition générale: «les phénomènes d'influence concernent les processus par lesquels les individus et les groupes façonnent, maintiennent, diffusent et modifient leurs modes de pensées et d'action lors d'interactions sociales directes ou symbolique " (p.7); ils rappellent le rôle fondamental de l'influence dans la constitution de la société, la cohésion, l'évolution et la pérennité des groupes, justifiant par là qu'un domaine scientifique puisse s'y intéresser. Ils reviennent alors sur les fondamentaux proprement dits : 1) les hypothèses de Leon Festinger parus dans un article de $1950^{4}$ sur la communication dans les groupes et ses fonctions, et dans lequel se lisent les principales problématiques du domaine: "le rôle capital de la divergence et de la communication dans les dynamiques d'influence sociale, l'importance de la pression sociale et des liens sociaux de dépendance qui unissent ou séparent la source et la cible de l'influence [...], et finalement l'importance des résistances à l'influence... » (p. 10). 2) Les expériences de Muzafer Sherif (1935) sur la normalisation ${ }^{5}$ qui ont mis en évidence le rôle des positions sociales des membres d'un groupe, de l'identité, et de la croyance au consensus comme gage de vérité, dans les processus d'influence. 3) Et enfin, les travaux de Solomon Asch ${ }^{6}$ sur la conformité, qui mettent en évidence le lien entre influence et rapport social à une majorité, paradigme dont dérivent dans l'ensemble les travaux sur l'influence sociale, dont la visée est d'éclairer les processus constitutifs de celle-ci. 
Ce sont ces processus qui sont présentés dans le deuxième chapitre, à travers six sections. Tout d'abord, les auteurs abordent l'imitation, selon ses deux principales orientations fonctionnelles, celle d'apprentissage par reproduction (imitation d'un modèle), et celle de facilitation de l'interaction. La première renvoie l'influence à un processus de type constructiviste, qui implique une activité d'interprétation de la cible quant au contenu de l'influence, et un ajustement du modèle au comportement de la cible. La deuxième renvoie l'influence à des effets qui découlent de la facilitation des interactions induits par l'imitation (l'imitation joue favorablement sur les impressions qu'un sujet imité peut avoir de l'imitateur).

9 Sont ensuite abordés les processus automatiques de l'influence sociale (qui échappent à la conscience), et en particulier l'ancrage (donnée qui, dans un discours, influence le jugement sur celui-ci, indépendamment de la ligne argumentative); le conditionnement évaluatif (report de la qualité d'un objet sur la perception et le jugement d'un autre objet); et l'amorçage (report de la qualité d'un objet sur les comportements).

Dans une troisième section, les auteurs s'arrêtent sur la recherche du consensus dans un groupe, et donc les modalités de celui-ci, la façon dont il se réalise, soulignant le rôle du consensus dans la quête de certitude.

11 La quatrième section est consacrée à la dépendance, qui offre un autre point de vue que celui du consensus pour expliquer qu'un individu puisse changer son jugement à propos d'un objet pour se rapprocher du jugement du groupe. C'est le degré de dépendance d'une source sur une cible qui est étudié, et donc les modalités de l'influence qui en découlent. Deux types d'influence sont mis en évidence: une influence de type informationnelle (accorder davantage de crédits aux réponses d'une source à une question qu'aux siennes propres), et une influence de type normative (qui découle du désir d'obtenir quelque avantage d'être associé au groupe). Il apparaît par ailleurs que cette partition de l'influence se double d'une autre qui renvoie à l'intensité de la conformité au groupe, variable selon trois niveaux: de complaisance, d'identification et d'intériorisation.

La cinquième section, intitulée "La saillance des normes ", interroge l'influence de certaines normes - normes descriptives (ce qui est fait communément) et normes injonctives (ce qui est communément approuvé ou sanctionné socialement) - sur le comportement des individus, et la propension de ceux-ci à se conformer à celles-là. D'où il apparaît que ce qui se joue à travers la conformité ou la non-conformité aux normes concerne l'identité des sujets. Et c'est en particulier ce lien entre conformité, influence et identité qui est au cœur de la sixième section : sont exposés ici les travaux qui se sont intéressés à l'influence sociale dans ses implications identitaires. En particulier, deux cadres théoriques sont mobilisés : la gestion des impressions, et les théories de l'identité sociale et de l'autocatégorisation. Le premier concerne «le processus par lequel un individu tente de contrôler l'impression qu'il donne aux autres » (p. 46), processus qui intervient dans l'influence, comme facteur fondamental de conformité ou de non-conformité (l'image que l'on veut donner de soi conduisant à se conformer ou à se distinguer / dévier du groupe). Le deuxième concerne le rôle de l'identité sociale (soit la représentation qu'a un individu de lui-même comme appartenant à un groupe et la valeur qu'il attribue à ces appartenances) sur l'influence sociale; il conduit à envisager l'influence selon que la source et la cible appartiennent ou non à un même groupe, et selon le niveau d'identité mobilisé en situation 
d'influence (comme partie prenante d'un «nous ", comme distinct des autres membres du groupe, comme distinct d'autres groupes, comme distinct d'autrui). D'où il ressort qu'un individu est davantage influencé par les autres membres de son groupe que par les membres d'un hors-groupe.

Pour finir, dans le prolongement des travaux s'appuyant sur les théories de l'identité sociale, les auteurs évoquent les travaux sur la stigmatisation sociale et les stratégies individuelles et collectives (reflet d'une influence de la stigmatisation sur les comportements des stigmatisés) mises en œuvre pour y faire face.

Le troisième chapitre, divisé en six sections, est consacré à une question fondamentale de la recherche dans le domaine de l'influence sociale, celle de l'influence des minorités et de l'innovation.

Les travaux de Serge Moscovici menés en $1976^{7}$ sont pris ici comme référence inaugurale d'un projet qui connaît encore aujourd'hui des développements, et auxquels les auteurs en particulier sont associés. On doit à Serge Moscovici d'avoir rompu avec le postulat jusque-là établi selon lequel l'influence d'une minorité dépendait de la position de la source par rapport à la majorité, c'est-à-dire des ressources psycho-sociales dont il disposait, ce qui supposait une logique hiérarchique prévalant dans les processus d'influence. Il s'agit donc dans ce chapitre de présenter les travaux qui traitent des processus engagés selon que la source d'influence renvoie à une minorité ou une majorité, et sur la capacité des minorités à porter une innovation et produire du changement. Les nombreux facteurs intervenant dans le processus d'influence sont mis en évidence: la consistance (diachronique et synchronique) comme principe fondamental de l'influence minoritaire, la flexibilité dans la défense d'une position (contre la rigidité), l'appartenance ou non de la source minoritaire au groupe cible de l'influence, les enjeux identitaires impliqués dans le rapport entre les cibles et la source minoritaire... Sont également abordés les différents niveaux d'effets de l'influence sur une cible (influence manifeste, publique, directe contre influence latente, privée, différée), selon que la source est minoritaire ou majoritaire ; l'effet d'influence, selon que la source minoritaire « déviante » fait partie du groupe ou n'en fait pas partie et la perception de cette appartenance selon le contexte; les formes de pensée de la cible induites par le fait que la source est reconnue comme majoritaire ou minoritaire; le traitement de l'information et l'influence selon que la cible se réfère à l'identité du groupe minoritaire source ou au contenu du message de la source (dissociation)... Ce chapitre met ainsi en évidence toute l'importance et la diversité du traitement cognitif dans les processus d'influence.

Enfin, partant du constat des disparités et du relatif manque de cohérence et de consistance des résultats de la recherche, les auteurs consacrent le quatrième et dernier chapitre aux travaux qui relèvent de perspectives intégratrices, de recherche d'explication et de mise en articulation du disparate, et ce en situant l'analyse à un niveau de généralité supérieur. Deux directions sont retenues :

- la première met l'accent sur les modalités de traitement (en profondeur, superficielle) de l'information (relative au contenu, à la source, au contexte, aux motivations de la cible) par une cible, et sur les implications de ce traitement en termes d'influence, avec l'hypothèse que «selon les circonstances, une majorité peut tout autant qu'une minorité induire une influence profonde » (p. 89) ;

- dans la seconde, ce sont les significations associées à la divergence entre une source et une cible concernant un objet - significations révélatrices d'un conflit qui sont au cœur de modèles explicatifs des processus d'influence. Dans cette 
perspective, la nature de la source (selon qu'elle est majoritaire ou minoritaire, qu'elle occupe ou non une position d'expert, et selon son appartenance à l'intragroupe ou au hors-groupe), croisée avec des tâches différenciées (tâches objectives non ambiguës, tâches d'aptitudes, tâches d'opinion, tâches non impliquantes) définissent le cadre d'élaboration par la source de conflits (épistémique ou relationnel) induisant des formes particulières d'influence. Cette orientation implique ici plus particulièrement les travaux des auteurs et la présentation vise à rendre compte des avancées réalisées depuis les années 90.

reprend une grande partie de celui-là avec un égal souci d'exhaustivité - inscrit dans le projet de la collection - et dont témoigne le nombre important de références, de cadres expérimentaux, d'hypothèses testées, de propositions théoriques, exposés ici. Mais l'exercice consistant à aller à l'essentiel en couvrant le mieux possible le champ considéré se fait aussi au détriment de la clarté, en raison d'une économie d'explications concernant les méthodes, les conditions d'expérimentation ou encore les controverses propres au champ. C'est d'autant plus marqué ici que le format est plus court que celui adopté en 1995. Ainsi, l'accumulation d'observations, de mises en tension de variables multiples pour saisir l'influence, définit un espace de savoir labyrinthique, en dépit de la rigueur de la construction du livre. Et les efforts de concision n'échappent pas toujours au sentiment que certaines propositions relèvent de la tautologie ; ainsi par exemple, l'hypothèse selon laquelle « l'influence des normes du groupe croît à mesure qu'augmente l'identification personnelle au groupe » (p. 55) appelle la question des critères d'évaluation de cette identification si ce ne sont les normes du groupe elles-mêmes, dans lesquelles l'individu se reconnaît.

19 Livre-repère, livre-mémo, en regard de la somme qu'il présente, il semble d'abord s'adresser aux acteurs du champ, en premier lieu les étudiants poursuivant des études en psychologie sociale, qui pourront y voir une carte détaillée d'un des domaines de la psychologie sociale. Il faut être quelque peu familier du domaine pour s'y retrouver avec aisance, et pour apprécier pleinement toutes les nuances des résultats qui découlent de variations parfois minimes dans les protocoles expérimentaux. Ne l'étant pas suffisamment, je ne suis guère en mesure de saisir, par exemple, la portée des évolutions et des avancées du domaine depuis le milieu des années 90 , et ne peut guère aller au-delà du constat que les auteurs y contribuent beaucoup à en juger le nombre de références à leurs propres travaux.

20 Un autre intérêt de cet ouvrage est assurément de donner à lire la complexité des mécanismes de l'influence sociale, l'indéfinie pluralité des paramètres qui interviennent dans sa dynamique, l'amplitude variable de son intensité ; à l'opposé 
d'une représentation ordinaire qui réduit souvent l'influence à un coup de force, à une forme de soumission-conformité (qui conduit parfois à faire de l'individu influencé une victime), les travaux ici présentés mettent en évidence - dans une perspectiviste cognitiviste - le rôle de la « cible » dans cette dynamique, rôle plus ou moins conscient, et qui témoigne des enjeux qui participent de cette dynamique : enjeux identitaires en premier lieu (personnel ou social), enjeu d'appartenance, enjeu de reconnaissance, enjeu de certitude et de connaissance...

21 Le chercheur en information-communication, pour qui la notion d'influence renvoie à des questions de construction discursive nouée à une intentionnalité, à des questions de réception et de représentations, d'usages de technologies, de circulation de l'information, de comportements induits par des contextes de communication, pourra trouver dans ces enjeux des repères sur lesquels porter son attention dans l'analyse de situations de communication d'influence (de l'intérêt, par exemple, qu'il y a à se faire influencer, à se laisser convaincre); il pourra aussi trouver dans ces processus complexes de l'influence une entrée d'analyse des pratiques sociales et communicationnelles pour y saisir ce qui s'y joue du point de vue de l'influence (et de la réciprocité de l'influence), les modalités ou les déterminants qui y sont activés volontairement ou non - à cette fin.

Mais si le lecteur est aussi praticien de l'influence, il pourrait bien être pris de vertige à la lecture de ce livre; il trouverait sans doute que la notion de "contexte de réception ", telle que la définit Philippe Breton ${ }^{8}$ pour aborder l'argumentation comme acte de communication, demeure bien réductrice du processus qui participe de l'art de convaincre ; et peut-être aussi trouverait-il son activité un peu vaine, quoique le livre puisse lui fournir matière à nourrir son action... «Pour le meilleur comme pour le pire». Cette dualité morale au cœur de la question de l'influence est d'ailleurs mentionnée par les auteurs. On regrettera cependant qu'elle ne fasse pas l'objet d'une incursion plus poussée du côté de l'éthique, d'autant que celle-ci est une préoccupation importante des acteurs du champ, repérable dans nombre d'ouvrages du domaine, préoccupation qui concerne en premier lieu les protocoles d'expérimentations, mais aussi les usages sociaux de ses résultats. On le regrette parce que dans ce domaine, et dès lors que l'ouvrage se donne à lire comme l'actualisation d'un travail de synthèse réalisé 22 ans auparavant, il aurait été intéressant de savoir si les positions avaient bougé, et intéressant de comprendre les enjeux de certaines recherches dont le rapport à l'actualité est manifeste.

\section{NOTES}

1. Beauvois J.-L. (2005). Les illusions libérales, individualisme et pouvoir social. Petit traité des grandes illusions, Grenoble, Presses universitaires de Grenoble.

2. Dubois N. (1994). La norme d'internalité et le libéralisme, Grenoble, Presses universitaires de Grenoble.

3. Watzlawick P. (1980). Le langage du changement, Paris, Seuil, p. 19. 
4. Festinger L. (1950). «Informal social communication ». In Psychological Review, vol. 57, n 5, p. 271-282.

5. Sherif M. (1935). " A study of some social factors in perception ». In Archives of Psychology, vol. $27, \mathrm{n}^{\circ} 187$, p. 23-46.

6. Asch S. E. (1956). «Studies on independence and conformity: a minority of one against a unanimous majority ». In Psychological Monographs: general and applied, vol. 70, nº 416, p. 1-70.

7. Moscovici S. (1976). Social influence and social change, London, Academic Press.

8. Breton P. (1996). L'argumentation dans la communication, Paris, La Découverte, p. 19.

\section{AUTEURS}

\section{THOMAS HELLER}

Univ. Lille, EA 4073 - GERiico -

Groupement d'Études et de Recherche Interdisciplinaire en Information et Communication F-59000 Lille, France

thomas.heller@univ-lille.fr 